Pediat. Res. 13: 1387-1393 (1979)

\title{
Index to Volume 13
}

Aborted SIDS 136

Acetylcarnitine 10

Acetylcholine 1052

Acetylcholinesterase 1217

Acid secretion 36

Acidosis, acute and perinatal 178

Acute rheumatic fever 16

Adams, F. H. 777

Adenosine deaminase deficiency 649

Adipose tissue 626

Adrenergic receptors 1024

Affinity chromatography 91

Agee, C. C. 31

Aiuti, F. 797

Akakoshi, I. 1134

Aldosterone 603, 755, 1379

Aleck, K. A. 1179

Allan, W. C. 1121

Allen, L. G. 184

Alpin, C. E. II 924

Alternative pathway of complement activation 641

Altman, Y. 803

Alvear, J. 215

Ambrose, S. 707

Ament, M. E. 36

Amino acids 65, 100, 236

Amino acid transport 854, 861, 1058

Aminoaciduria, neonatal 854, 861

Aminophylline 1330

Ammirati, P. 797

Ammonia 117

Amniotic fluid 654

c-AMP 884

Amylase 1024

Anand, R. S. 783

Anderson, A. 788

Anderson, D. W. 208

Andres, J. M. 1290

Angiotensin 603

Anthropometry 170

Antibody response 987

Antidiuretic hormone 257,606, 841, 884

Aortic pressure 1271

Aortic stenosis, supraval 121

Apnea 1144, 1271

Arashima, S. 1134

Arginase deficiency 827

Arginine vasopressin 257, 606, 841

Argiolu, F. 812

Arnold, M. 215

Arterial-alveolar differences 1167

Arterial blood flow 1271

Arysulfatase B 1203

Asphyxia 1

Ataxis telangiectasia 803

Atkin, B. M. 38, 109, 1101

Atropine 1271

Auricchio, S. 1255

Autonomic nervous system 139

Averill, D. R. 52
Bacterial infection 1093

Baehner, R. L. 769

Bakay, B. 1365

Ballowitz, L. 1307

Balsan, S. 973

Banker, B. Q. 109

Barbero, G. J. 760

Bard, H. 167

Barnard, D. R. 1330

Barnes, A. 265

Barnett, D. R. 1137

Barrett, C. T. 752, 1336

Barrow, R. E. 924

Basement membranes 774

Basner, R. 1316

Bass, N. H. 26

Basuyau, J.-P. 647

Battaglia, F. C. 117

Becker, N. 1141

Beckerman, R. C. 1137

Bell, E. F. 1012

Bellanti, J. A. 672

Beran, A. V. 821

Beratis, N. G. 206

Bercu, B. B. 142,246

Berenson, G. S. 170

Berkel, A. I. 16

Berlin, R. D. 1058

Bidot-López, P. 1002

Bilirubin 1326

Binswanger, U. 963

Biological half-life 635

Biopsy 1240

Biotin 746, 1275

Birdwell, R. L. 611

Birth 611, 1285

Björkstén, B. 737, 1341

Bland, R. D. 1037

Blandin-Savoja, F. 21

Blastocyst 937

Bleasdale, J. E. 1182

Blessing-Moore, J. 1030

Blood 752, 1280, 1361

Blood-brain 161

Blood, cord 148

Boat, T. F. 1129

Body fat 7, 170

Body weight 104

Boffey, P. M. 726

Bonderman, P. 1326

Bone 1105

Bossen, A. 1030

Bove, K. E. 121

Bowes, J. E. 716

Boxer, L. A. 769

Boxerkaum, B. 1085

Boychuk, R. B. 875

Boyden's chamber method 148

Boyer, K. M. 1015

Braddock, M. E. 1024

Bradycardia 1052
Brain 26, 585, 1141

Brain carbohydrate metabolism 52

Branched-chain amino acids 889

Brand, N. 1179

Brandt, N. J. 977

Brazy, J. 599

Brent, R. L. 1211

Bressack, M. A. 1037

Bright, M. J. 31

Brooke, O. G. 215

Brorkowsky, W. 649

Brumley, G. 599

Brunelle, P. 647

Bucciarelli, R. L. 178

Buch, M. 10, 626

Buchino, J. J. 121

Buist, N. R. M. 109

Bumpers, B. 704

Bunjamin, A. 1307

Burghen, G. A. 870

Businco, L. 797

Butler, J. D. 1350

Byrne, W. J. 36

Cabal, L. 1163

Cadmium 197

Cahill, F. 265

Calcagno, P. L. 774, 1379

Calcitonin 1

Calcium 1, 963, 1262

Calvani, M. 797

Cao, A. 812

Carbohydrate 1065

Carcinoembryonic antigen 91

Cardiac dimensions 910

Cardiac function 910

Cardiac output 910,1112

Cardiac sympathetic nerves 136

Cardiopuulmonary disease 1163

Cardiovascular function 1112

Carnitine 10

Carper, J. 720

Carrier detection 1101

Carter, J. N. 211

Cartilage 904, 1222, 1266

Cassiman, J. J. 1384

Cates, D. 875, 982

Cathelineau, L. 207

Catterton, W. Z. 104, 1144

Cavalli-Sforza, L. L. 1030

Cederbaum, S. D. 827

Cell aging 1231

Cell culture 764, 1097

T-Cell defects 797

T-Cell function 803

Cellular immunity 16

Cellular immunodeficiency 803

Cerebral vascular bed 178

Cerebrospinal fluid 26

Ceruloplasmin 845

Champion, J. G. 1015 
Chan, G. M. 121, 1383

Chan, J. C. M. 591

Chan, W.-Y. 197, 204

Char, V. C. 1018

Charache, S. 851

Chemotactic function 148

Cherry, J. D. 1015

Chesney, R. W. 854, 861

Chest distortion 982

Chez, R. A. 788

Cholesterol synthesis 1121

Choline 635

Cholinergic innervation 1217

Chondrocyte 904, 1266

Chorionic somatomammotropin 194

Chow, D. M. 1356

Christensen, M. B. 1371

Christie, A. 1301

Chromosome abnormalities 1247

Chronic granulomatous disease 769

Chronic hemodialysis in children 591

Chronic pancreatitis 1030

Chronic reserpine administration 1024

Chymotrypsin 969

Cigarette smoke 987

Ciliary activity 31

Ciliary dyskinesia factor 729

Circulation 900

Citrate synthase 38

Citrullinemia 1134

Clark, A. 44

Cleavage 937

Clopath, P. 845

Clutario, B. C. 1285

$\mathrm{CO}_{2}$ 982, 1065

Cohen, M. I. 644

Cohn fractionation 845

Collagen 904, 1222, 1266

Collagen, metabolism 774

Colostrum, human 737

Complement 1043, 1093, 1341

Complement, synthesis of 188

Concentrating capacity 884

Congenital renal alkalosis 1379

Conjoined twins 924

Constant positive pressure breathing 1167

Continuous distending airway pressure 261

Continuous distending pressure 250

Continuous intravascular electrode 821

Cooper, C. 1024

Cooper, R. B. 987

Copper 197, 845, 959, 1222, 1280

Corket, A. J. S. 1167

Cord blood 641, 845

Cortisol 96

Corrigan, Jr., J. J. 48

Cortisol 1097

Cotton, R. C. 1211

Cotton, R. G. H. 1150

Courtney, J. D. 1167

Cousins, L. 1

Cowett, R. M. 594

Clq 1043

Creasy, R. K. 1089

Creatinine 1012

Crenshaw, Jr., C. 599

Crying, 900

Csaba, I. 817

Cultured glia 1121

Cystic fibrosis 31, 131, 729, 764, 834, 1030 , $1129,1137,1156,1160,1371,1384$
Cystine 1350

Cystinosis 1350

Cystinuria 827

Daabees, T. T. 894

Dahms, W. T. 7

D'Amelio, R. 797

Dancis, J. 236

Danks, D. M. 1150

Das, M. 632

Davi, M. 982

Davidson, G. 1077

Davis, C. A. 1043

Dearborn, D. G. 1085

de Groot, I. 1082

de Leon, L. B. 1125

De Menibus, C. H. 647

De Meyer, R. 1065

Demko, C. A. 1085

De Ritis, G. 1255

Dermatan sulfate 1203

Deseda-Tous, J. E. 1015

Desjeux, J. F. 1240

Desnick, R. J. 1179, 1294

Developmental hepatology 1290

De Virgiliis, S. 812

De Witt, D. P. 1326

DeWolfe, M. S. 959

Diabetes 1, 752, 1323

Diaphragm configuration 250

Diastrophic dwarfism 904

Diazepam 992

$\mathrm{C}_{6}-\mathrm{C}_{10}$-Dicarboxylic acid 977

Diet 10

Dietary composition 7

Digestion 1018

Digoxin 26

Dihydropterine reductase (DHPR) 1150

24,25-Dihydroxyvitamin D 973

Dimmick, J. E. 1323

Dinari, G. 644

Dinwiddie, R. 900

Di Paolo, R. 845

2,3-Diphosphoglycerate 167

Dizio, D. 774

DNA 1125

Dobutamine 1006

Dodge, M. 1

Dolfin, Z. 803

Donahoe, P. K. 142, 246

Donckerwolke, R. A. 1177

Dönges, H. 152

Dopamine 1006

Down's syndrome 803

Downing, C. J. 916

Driscoll, D. J. 1006

Dukin, J. W. 1375

Duc, G. 963

Duchenne muscular dystrophy 152

Duckworth, W. C. 870

Dudley, J. P. 1015

Dumas, R. 1105

Dumontier, A. M. 1240

Dupont, B. 1356

Durie, B. G. M. 1137

E. coli 737

E. coli endotoxin 1290

Echinocyte formation 152

Effros, R. M. 841

Eitzman, D. V. 178
Elastase 969

Elders, M. J. 96

Electrocardiogram 139

Electrolyte balance 817

Electron spin resonance spectroscopy 845

Embryo 937

Endochondral growth plate 904, 1266

Endotoxin activity 48

Endotracheal tube leakage 60

Energy 215

Eng, G. 591

Enolase 1231

Erenberg, A. 230

Enzyme inhibition 889

Epidermal growth factor (EGF) 104

Epithelium, ciliated 131

Epstein, M. A. F. 136, 139

Epstein, R. A. 136, 139

Ergocalciferol 973

Ertl, T. 817

Erythrocytes 649

Erythrocytes, rheology of 152

Erythropoiesis 851

Escobedo, M. B. 104

Euler, A. R. 36

Evaporation through skin 1072

Exchange transfusion 963

Experimental meningitis 52

Factor B 641

Faiman, C. 211

Fallstrom, S. P. 26

Familial renal iminoglycinuria 65

Fat 615

Fatty acid oxidation 10

Favism 812

Feather, M. S. 760

Feig, S. A. 632

Feigal, R. J. 764

Feline, 1203, 1294

Feline $\mathrm{GM}_{2}$ gangliosidosis 916

Fenton,L. J. 641

Fernandes, J. 225

Ferritin, 143

Fetal erythropoiesis 1231

Fetal gonadotropins 246

Fetal heart 1052

$\alpha$-Fetoprotein 1290

Fetus 100, 104, 194, 230, 599, 606, 623, 752 , $851,910,997,1018,1097,1125,1172$, $1211,1255,1319,1330,1375$

Fibroblasts $38,197,764,1101,1384$

Fibroplasts, human 127

Figura, K. 1316

Finch, C. A. 1010

Firgaira, F. 1150

Fischer, J. A. 963

Fiser, W. P. 1

Fisher, D. A. 1, 257, 603, 606, 841

Fluharty, A. L. 1179

Forbes, G. B. 1382

Forristal, J. 1043

Forstner, G. G. 1077

Fox, W. W. 60, 900

Franchi, F. 797

Francke, U. 1365

Frankfater, A. 1217

Frantz, I. D. III 250, 261

Frau, F. 812

Freeman, G. 1015

Frerichs, R. R. 170 
Friedman, W. F. 910

Friedman, Z. 932

Frolich, J. C. 932

Fructose 1018

Functional residual capacity 60

Gabridge, M. G. 31

Gaddis, L. 937

Gadot, N. 803

Gahr, M. 1231

Gaines, J. A. 641

Galactosyltransferase, 729

Ganguli, S. 783

Garabedian, M. 973

Garnica, A. D. 197

Gartside, P. S. 208

Garrett, R. 1156

Geering, J.-M. 742

Genetic disease 649

Gentamicin 16

Gerard, P. 1065

Gestation 1163

Giant cell induction 1290

Gibbs, G. E. 1371

Gibson, R. S. 959

Gilden, C. 1336

Gillette P. C. 1006

Girard, F. 221

Györy, E. 817

Glass, A. R. 7

$\alpha_{10}$-Gliadin 1255

Glomerular filtration rate 1012

Glossel, J. 1316

Glucagon 870

Gluck, L. 635

Gluckman, P. D. 194

Glucocorticoids 96, 997

Gluconeogenesis 109, 175, 594

Glucose 100, 117, 241, 585, 752, 1018, 1065, 1375

Glucose endogenous 594

Glucose oxidase 769

Glucose-6-phosphatase deficiency 225

Glucose-6-phosphate dehydrogenase 1361

Glucose-6-phosphatidehydrogenase 1231

Glucose transport 1129, 1240

Glueck, C. J. 208, 1082

$\gamma$-Glutamyl transferase 1058

Glutaric aciduria 977

Glutaryl-CoA dehydrogenase deficiency 977

Glycine 65

Glycine cleavage system 889

Glycine transport 127

Glycogen 1065, 1319

Glycoproteins 834, 1211

Glycosaminoglycan 96

Gobran, L. I. 623

Godard, C. 742

Goetzman, B. W. 942

Goldman, M. D. 250

Gootman, N. 1271

Gootman, P. M. 1271

Gothefors, L. 737

Gourmelen, M. 221

G6 PDd activity 812

Gram-negative septicemia 48

Gramenzi, F. 1255

Granulocytes, 148

Grasset, E. 1240

Gray, A. 697

Gray, M. E. 104
Green, J. R. 1089

Gregersen, N. 977, 1227

Groshong, T. D. 928

Gross, I. 623, 1319

Growth 215, 221, 225

Growth hormone 194

Growth retardation, intrauterine 100

Grumbach, M. G. 194

Guillozo, H. 973

Guinea pigs 167

Gutcher, G. G. 997

Häberle, D. 884

Haddad, G. G. 136, 139

Haemophilus influenzae 52

Hagemeijer, A. 1247

Hahlen, K. 1247

Hahn, P. 10

Hahn, T. 803

Hair, 959

Hamosh, M. 615

Handzel, Z. T. 803

Hanefeld, F. 1307

Hanley, E. J. 1326

Hannemann, R. E. 1326

Hansen, T. N. 1167

Hara, K. 1222

Hardy, K. J. 265

Harrison, H. R. 188

Harsha, D. W. 170

Hartley, C. J. 1006

Haskins, M. E. 1203, 1294

Hathaway, P. J. 851

Hathaway, W. E. 1330

Hayes, H. 671

Heart, 161, 184, 1006

Heart rate 1052,1271

Heggie, A. D. 937

Helium rebreathing 60

Hemagglutinating antibodies 156

Hemodialysis 1105

Hemoglobin, analysis 143

Hemoglobin C 1109

Hemoglobin S 1109

Hemolytic crises 812

Hemorrhage 606

Henderson, N. S. 31

Henderson T. 632

Hennings, G. 1150

Heparin 834

Hepatic excretory function 1002

Hepatic fructose-1,6-diphosphatase deficiency 1361

Hepatic gamma glutamyl transpeptidase 644

Hepatic glycoprotein receptors, 916

Hepatic UDP-bilirubin glucuronyl transferase 644

Herscowitz, H. B. 987

Hereditary nephritis 774

Hering-Breuer reflex 261

$\mathrm{Gd}^{\text {Mediterranean }}$ heteroxzygous females 812

Heubi, J. E. 781

$\beta$-Hexosaminidase, 916

Heyman, M. 1240

High density lipoprotein cholesterol 208

Hill, I. D. 733

Hillman, R. E. 127

Hinds, T. R. 131

Hinman, A. R. 689, 721

Hirschhorn, K. 206

Hirschhorn, R. 649
Histamine 942

L-Histidine 845

Histochemistry 1266

HMG-CoA reductase 1121

Hodgman, J. E. 1163

Hodson, W. A. 654

Hollister, D. W. 904

Holzman, B. H. 1112

Holzman, I. R. 117

Homocyst(e)ine 1141

Homocystinuric 1141

Hon, E. H. 1163

Honig, G. R. 1109

Hopfer, U. 1129

Horacek, I. 265

Horton, W. A. 904, 1266

Host defense 1093

Howard, G. 184

Hsia, Y. E. 746, 1002, 1280

Huang, P. S. 611

Hudson, B. G. 1211

Hug, G. 44

Hughes, E. R. 96

Human milk 969

Human newborns 626

Hung, C. H. 1211

Hunt, J. A. 1323

Hunter, G. T. 698

Hurler syndrome 1294

Huxtable, R. F. 821

Hyaline membrane disease 654

Hyaline membrane disease 1167

25-Hydroxycholecalciferol 1105

Hydroxylase activity 21

Hydroxylysine glycosides, urinary 774

Hyperammonemia 807, 827

Hyperargininemia 827

Hyperbilirubinemia 1307

Hypercalcemia, infantile 121

Hyperglycinemia, nonketonic 127

Hyperoxia 875

Hyperphenylalaninemia 21

Hyperplasia 1379

Hypertension 992

Hypocalcemia 1, 647,963

Hydrocephalus 26

Hypogammaglobulinemia 181

Hypoglycemia 175

Hyponatremia 257, 817

Hypothalmus 246

Hypoxanthine guanine phosphorbosyl transferase 1365

Hypoxia 875

I-cell disease 1350

$\alpha$-L-Iduronidase 1294

IgA plasma cells 733

IgG 1043

IgG subclasses 181

Ikegami, M. 777

Imanishi, Y. 1134

Immune dysfunction 632

Immunocompetence 649

Immunodeficiencies 797

Immunofluorescence studies $\mathbf{7 3 3}$

Immunologic maturation 987

Immunologic maturity 733

Immuno-suppression 987

Infant 1043

Infant respiration 250

Influenza 44 
Influenza surveillance 1015

Insensible water loss 1072

Inspiratory and expiratory duration 261

Insulin 221, 594, 752, 870, 1375

Interferon 937

Intestine 1255

Intracellular compartment 161

Intralipid 188

Intrapulmonary foam 1285

Intrauterine growth retardation 175

Aug- $\mathrm{Ag} / \mathrm{AgCl}$ Intravascular electrode 821

Intravascular saline infusions 1037

Inulin 26, 1012

lodine 959

Iron 143

Iron deficiency 1010

Islet cell antibody 1323

Ismail, G. 769

Isoproterenol 760, 1006

Itabashi, H. 1179

Iwashima, A. 1361

Jackson, I. M. D. 142, 246

Jansen, H. 220

Jansen, T. C. 225

Jaundice 1326

Jax, D. K. 854,861

Jejunum 733, 1240

Jessop, W. J. E. 1237

Jezyk, P. F. 1203, 1294

Joke, A. 635

Johnston, J. M. 1182

Jones, C. M. 851

Jonxis, J. H. P. 1072

Joppich, R. 884

Josso, N. 142

Kaback, M. M. 1179

Kamoun, P. 207

Kanfer, J. N. 845

Kang, E. S. 870, 946

Kang, S.-S. 1141

Kaplan, S. A. 752, 1336

Kaplan, S.L. 194

Karzon, D. T. 714

Katz, J. B. 139

Kelly, J. C. 127

Kelly, K. A. 1082

Kennedy, R. L. 230

Kenny, J. D. 1167

Keresztes-Nagy, S. 1217

$\alpha$-Ketoglutarate decarboxylase, 928

$\alpha$-Ketoglutarate dehydrogenase 928

Ketone bodies 10, 585

Ketosis 977

Ketotic hyperglycinemia 889

Khon, S. 729

Khoury, P. 1082

Kidney, 65, 257, 884, 1125, 1141, 1172, 1211

Kidneys, 161

Kiemann, U. 884

Kiernat, J. F. 48

Kihara, H. 1179

Kilgore, B. S. 96

Kinugasa, A. 1361

Kinky hair disease 1222

Klein, D. C. 788

Klein, U. 1316

Klimov, A. N. 208

Kline, J. J. 928

Kniest syndrome 1266
Kochen, J. A. 644

Kølvraa, S. 889

Korman, P. 1156

Kovar, I. 1144

Kraus, H. 585

Krause, P. J. 1015

Kresse, H. 1316

Krongrad, E. 136, 139

Krutzik, S. R. 1

Kuchenbrod, P. J. 1085

Kung, M.-P. 916

Kunke, K. 188

Kurlandsky, L. E. 1093

Kuroda, Y. 928

Kusunoki, T. 91, 1361

Kwashiorkor 156

LaBadie, G. V. 206

Labal, M. L. 1160

LaBrecque, D. R. 1002

Lactate 117

Lactic acid 1065

Lactic acidemia 928

Lactic acidosis $38,109,977,1101$

Lacticaciduria 225

Lactose 1018

Lancaster D. 161

Laryngeal chemoreflex 1144

Laser Light-scattering spectroscopy 131

Laskarzewski, P. 1082

Lawson, E. E. 611

Lasley, L. 65

Law, H. S. 139

Lead 644

Leake, R. D. 36, 257, 841

Lecithin/sphingomyelin ratio 623

Lee, J. A. 96

Leigh's disease 109

Leiter, A. B. 109

Lenard, H. G. 152

Lesch-Nyhan syndrome 1365

Lestradet H. 1240

Letarte, J. 807

Leucocytes, cystinotic human 1058

Leukemia, childhood 1247

Leung, C. C. K. 1211

Levamisole (Ketrax) 1237

Levin, S. 803

Levine, L. S. 755, 1356

Levy, M. E. 708

Lewis, R. M. 1006

LGH 221

Lietz, L. 1307

Lim, G. 1323

Lindberg T. 969

Lingual lipase 615

Linnemann, Jr., C. C. 44

Lipid 932

Lipid research clinics 208

Lipomal disorders 1203, 1294

Lipoprotein lipase activity 225

Liposomes 769

Liver 44, 161, 997, 1058, 1141

Livtchak, M. J. 208

Llanos, A. J. 1089

Long QT syndrome 136

Lorenzen, F. 1356

Lott, I. T. 845

Lou, M. F. 774

Low birthweight infants 1012, 1072

Low-density lipoprotein cholesterol 208
Low-V்A/Q́c units 1167

Lowe, C. U. 703

Lubin, A. H. 834

Luchtel, D. L. 654

Luciani, J.-C. 1105

Lucky, A. W. 1280

Lundborg, P. 26

Lung, 104, 161, 230, 611, 654, 729, 777, 997, $1037,1167,1285,1307,1330$

Lung lavage 599, 623

Lung maturation, fetal 1097

Lung phospholipids 635

Lung surfactant 623

Lung volume 261

Lymphocytes, 38, 649, 1101, 1237

Lysine 1134

Lysomal disorders 1203

Lysosomal enzyme replacement 916

MacCallum, M. 982

MacDonald, P. C. 1182

Mackler, B. 1010

$\alpha_{2}$-Macroglobulin 1030, 1384

Macrophages 188

Maffei, H. V. L. 733

Magracheva, E. J. 208

Magnetometers 250

Mahr, C. 699

Malignant hyperalaninemia 1150

Mallet, E. 647, 973

Malnutrition 156, 1237

Maltose 1018

Manganesese 959

Maniscalco, W. M. 1319

Marelyn, E. 265

Maigolis, C. Z. 1047

Marino, P. A. 623

Marteaux-Lamy syndrome 1203

Martinez, A. M. 1024, 1156

Martinez, J. R. 760, 1024, 1156

Mason, R. G. 1109

Maternofetal transport 100

Matson, J. R. 1172

Matsuda, I. 1134

Matsumoto, S. 148

Maurer, Jr. M. 1052

Mawhinney, T. P. 760

Mayer, G. 884

Mazique, E. C. 710

Mazza, N.M. 136, 139

McCubbin, J. A. 184

McCullough, R. A. 916

McDonough, S. K. 1294

McGowan, A. R. 1304

McGraw, C. A. 781

Mc Mann, B. J. 1125

Mc Namara, H, 644

McNatt, M. L. 96

McVie, R. 755

Meador, S. 96

Mehlhorn, D. 121

Meis, P. J. 36

Mellies, M. J. 1082

Mellins, R. B. 136, 139

Melnick, V. L. 672, 722

Membrane flexibility 152

Menkes Kinky hair disease 197, 1280

Mental retardation 109

2-Mercaptoethanol 1141

Meric, N. 16

Merrill, R. E. 1298 
Metabolic disorder 1222

Metabolic rate 215, 1072

Metabolism 241

Metachromatic leukodystrophy 1307

Metallothionein 197

B-Methylcrotonyl CoA carboxylase 1275

Methylxanthines 1121

Meunier, P.-J. 1105

Meves, H. 1231

Military clinic 1047

Milk formulas 10

Milk, human 143

Miller, L. R. 1010

Mills, E. M. 1341

Milner, G. R. 161

Milner, R. D. G. 161

Milstein, J. M. 942

Milunsky, A. 845

Mlekusch, W. 1316

Möhlen, K.-H. 236

Mononuclear cell metabolism 632

Moore, E. S. 1125

Mims, L. C. 241

Mitchell, R. A. 945

Mitochondria 764

Mitochondrial metabolism 1227

Miyazaki, S. 1222

Morikawa, Y. 142, 246

Morisi G. 1255

Morrison, J. A. 1082

Mortimer, E. A. 684

Moxon, E. R. 52

Mucolipidosis 1350

Mucopolysaccharides 1307

Mucopolysaccharidosis 1203, 1294

Mucosulfatidosis 1316

Müllerian inhibiting substance 246

Muntean, W. 1262

Murray, R. A. 1015

Murphy, J. H. 654

Mutz, I. D. 1262

Nagata, N. 1134

Nagle, R. B. 188

Nagy, E. C. 729

Nakayama, M. 148

Nathanielsz, P. W. 783

Németh, M. 817

Neonatal 632

Neonatal hepatitis 1294

Neonate 178, 585, 963, 1093, 1285, 1326

Neufeld, N. D. 752

Neuroblastoma tissue 91

Neurohypophyis 841

Neuropathies 591

Neutrophil, chemotaxia 737

Neutrophiles 1058

New, M. I. 755, 1356

Newborn 1, 36, 241, 257, 595, 615, 632, 641,

$742,841,884,900,910,932,942,959,987$,

$992,1037,1065,1163,1262,1307,1341$

Newborn, J. L. 1047

Newborn lambs 1144

Newborn testes 246

Newman, S. H. 705

Nevin, G. B. 1078

Nevin, N. C. 1078

Nguyen, T. M. 973

Nickerson, J. M. 31

Nissinen, E. 1365

Nitzan, M. 100
Nocturnal gastric drip feeding 225

Nogami, H. 1222

Nonlymphocytic leukemia 1247

Nonprescription cold remedies 1015

Nonspecific cross reacting antigen 91

Norman, M. E. 181

Novak, M. 10, 626

Nutrition, infant 143

Nutrition, parenteral 894

Nyhan, W. L. 928, 1365

Obesity 170

Occorsio, P. 1255

$\mathrm{O}_{2}-\mathrm{CO}_{2}$ diagram 1167

Ocampo, M. 1125

Odell, W. D. 1

Oh, W. 175, 594, 1012

Okken, A. 1072

Olusi, S. O. 156, 1237

Ontogeny 854,861

Oohira, A. 1222

Organ culture 1319

Organic acidemias/urias 1227

Orloff, S. 100

Ornithin 221

Orotic acid 807

Ornithine transcarbamylase 007

Otto, E. F. 127

Osteomalacia 1105

Ouellet, R. 807

Outpatient statistics 1015

Ovum 937

Oxidant stress 1097

100\% Oxygen 1167

Oxygen affinity 851

Oxygen tension 1097

\section{P50 167}

Palmas, C. 812

Palmer, S. 654

Palmetic acid 635

Palmitic acid 752

Pang, S. 1356

PAPase 230

Para-amino hippurate 1125

Parathormone 1

Parathyroid hormone 963

Parotid gland 1024

Parsons, M. 1030

Partin, J. C. 781

Partin, J. S. 868

Patrick, A. D. 1058

Patterson, D.F. 1203, 1294

Payne, C. M. 188

Pearson, R. D. 834

Pentagastrin 36

Peptic-tryptic-cotazyme (PTC) digest 1255

Perfusion 236

Person, R. 1010

Petek, W. 1262

Phagocytosis 737

Pham-Huu-Trung, M. T. 221

Phenylalamine 1010

Phenylalanine 21, 1150

Phenylalamine hydrorylase 1010

Phenyldiguanide 1271

Phenylketonuria 21

Phenylketonuria 1150

Philipps, A. F. 117, 1375

Phosphate ${ }^{32} \mathrm{P} 635$
Phosphatidylcholine 599, 623, 635, 997

Phosphoenol pyruvate carboxykinase 38

Phosphoenolpyruvate carboxykinase 175, 1101

Phosphofructokinase 585, 1231

Phospholipids 777, 1330

Phosphoribosylpyrophosphate 649

Phototherapy 1307

Pietrzyk, J. J. 879

Pilocarpine 760

Pitcher-Wilmott, R. 900

Pivetta, O. H. 1160

Placenta 236, 851

Placental metabolism 117

Plasma 1141

Plasma adenosine 3'5' 647

Plasma clearance 916

Plastic embedding 1266

Platelet function 1345

Pollack, M. 1356

Pollak, A. 175

Polymyxin B 48

Positive end-expiratory pressure 1112

Potassium 1024

Pregnancy 1323

Premature infants 817

Premature rabbits 635

Pressure-volume characteristics 777

Preterm infant 215, 875

Price, P. 206

Progressive intrahepatic cholestasis 1002

Problem-oriented record system 1047

Prolactin 194

Proline 65

Properdin 641, 1043, 1341

Propionic acidemia 746, 1276

Propionyl CoA carboxylase 746, 1276

Prostaglandins 932, 1345

Protease 1384

protease inhibitors 969

Protein osmotic pressure 1037

Protein content 760

Protein kinase 626

Protein, wheat 1255

Proteinases 1030

Proteoglycan 904, 1222, 1266

Prothrombin 1262

Proximal renal tubular acidosis 109

Prueitt, J. L. 654

Pseudohypothyroidism 647

Puberty 7

Pulmonary compliance 1112

Pulmonary edema 1037

Pulmonary function 924, 1112 .

Pulmonary lavage 760

Pulmonary surfactant 611,1330

Pulmonary vasodilutor action, 942

Purine metabolism 649, 1365

Futrescine 1137

Pyruvate carboxylase 38, 109, 928, 1101

Pyruvate carboxylase deficiency $38,109,1101$

Pyruvate dehydrogenase 585, 928

Pyruvate kinase 632

QT interval 136, 139

Quie, P. G. 1341

Quiet sleep 136

Qureshi, I. A. 807

Rabbit 241

Rabbits 167 
Rabier, D. 207

Raghavan, S. S. 845

Rattazzi, M. C. 916

Raye, J. R. 1375

Reem, G. H. 649

Reflex bradycardia 184

REM 136

REM and quiet sleep 139

Rembold, H. 1150

Renal function 742

Renal hypertrophy, compensatory 1125

Renal osteodystrophy 1105

Renal transport 854, 861

Renin 603, 606, 742, 755

Renin-angiotensin-aldosterone system 817

Rennert, O. M. 197, 204

Reppert, S. M. 788

Respiration 900

Respiratory distress syndrome $623,777,900$, 1097

Rey, F. 21

Rey, J., 21

Reyes, F. I. 211

Reye's Syndrome 44

Reye's syndrome survivors 870

Rheumatic carditis 16

Rhodes, M. L. 230

Riboflavin 1307

Richardson, C. J. 924

Rickets 973

Riely, C. A. 1002

Rigatto, H. 875, 982

Right atria 1217

Rimoin, D. L. 904, 1266

Rispens, P. 1072

Robertson, Jr. W. C. 845

Robillard, J. E. 606, 1172

Robinson, B. 869

Romeo, G. 1030

Romero, T. E. 910

Rooney, S. A. 623

Rosanelli, K. 1262

Rose, J. C. 1089

Rosen, F. 181

Rosenberg, L. E. 746, 1275

Rosenblum, M. G. 1137

Rudolph, A. J. 1167

Rudolph, A. M. 194, 1018

Russell, D. H. 1137

Saarinen, U. M. 143

Sabin, A. B. 674, 723

Salicylate intoxication 44

Saliva 755

Saliva, submaxillary 1156

Sandhoff disease 916

Sankaran, K. 875, 982

Sanna, G. 812

Saralasin acetate 603

Saturated phosphatidyl choline 1097

Sawada, T. 91

Scarpelli, E. M. 1112, 1285

Schaeffer, A. 44

Schedewie, H. K. I

Schegel, R. J. 688

Schieken, R. M. 992

Schiff, G. M. 44

Schleman, M. 1271

Schlesinger, P. 1150

Schneider, H. 236

Schreiner, R. J. 1326
Schröter, W. 152, 1231

Schubert, W. K. 781

Schulman, J. D. 100, 1058

Schur, P. H. 181

Schwartz, A. 1006

Schwartz, J. G. 60, 900

Schwartz, R. 175, 594

Schwenzer, K. S. 870

Scoggins, B. A. 265

Scriver, C. R. 65

Scully, K. J. 746

Selstram, V. 1144

Séron-Ferré, M. 1089

Serum 181

Serum ciliotoxic activity 1160

Serum effects 1129

Serum factor 729

Serum lipids 225

Seshia, M. M. K. 875

Sessions, C. 1172

Sevanian, A. 752, 1336

Sexson, W. R. 104

Shaffer, T. H. 60, 900

Shapiro, B. L. 764

Shapiro, L. J. 1179

Shapiro, M. 167

Shaw, K. N. F. 827

Sheehan, T. J. 1047

Sheep 1172, 1375

Sheldon, G. 599

Shestor, D. B. 208

Shigezawa, G. Y. 821

Shiner, M. 733

Shinozuka S. 1134

Shoroye, A. 1237

Short-chain monocarboxylic acids 977, 1227

Short circuit rat jejunum bioassay 1371

Siassi, B. 1163

Sickle cell syndrome 1109

Sidenvall, R. 737

Siegel, S. R. 603, 841

Siimes, M. A. 143

Silano, V. 1255

Silberberg, R. 904

Silverman, J. 777

Simmons, M. A. 1330

Sinha, S. N. 1217

Skala, J. P. 626

Skin reflectance, black and white 1326

Sleep state 982,1163

Sloate, R. 711

Smit, E. M. E. 1247

Smith, A. L. 52

Smith, B. T. 1097

Smith, Jr. F. G. 606, 1172

Smith, J. B. 184

Snodgrass, P. J. 827

Sodium 127, 742

Solomon, S. S. 870

Somatomedin 96, 194

Sordelli, D. O. 1160

Source-oriented record system 1047

Soybean oil emulsion 188

Sparse-fur (spf) gene 807

Specific dynamic action 215

Spector, E. B. 827

Speight, Jr. J. T. 712

Sperling, M. A. 783

Sperm translation-cessation time 1160

Spermidine 1137

Stahlman, M. T. 104, 1144
Standaert, T. A. 654

Standard, R. L. 724

Staphylococcus aureus 156

Stark, A. R. 250, 261

Stegink, L. D. 894

Steichen, J. J. 121

Stein, E. A. 208

Steiner, P. M. 208

Stern, C. M. M. 792

Stevens, R. L. 1179

Stonestreet, B. S. 175, 1012

Stool 1280

Strunk, R. C. 188, 641

Stuart, M. J. 1345

Stumpf, B. 585

Sturgess, J. M. 729

Stuttgen, G. 1307

Styne, D. M. 194

Sucrose 1018

Sudden infant death syndrome 136,139

Sugarman, G. I. 827

Sugimoto, T. 91

Sulyok, E. 817

Sundell, H. W. 104, 1144

Surface tension 777

Surfactant 230, 654, 777, 1319

Surfactant production 752

Susa, J. B. 175, 594

Swallowing 1144

Sweetman, L. 928, 1365

Swerdloff, R. S. 7

Swift, A. J. 1093

Tachypnea 1271

Taeusch, Jr. H. W. 611

Tanswell, A. K. 1097

Taurine 854, 861

Taussig, L. 1076

Taussig, L. M. 1137

Tay-Sachs disease 916

Tényi, I. 817

Teratology 1211

Tetrahydrobiopterin $\left(\mathrm{BH}_{4}\right) 1150$

$\alpha$-Thalassemia 1109

$\beta$-Thalassemia 1345

Thermostability 746

Thomassen, M. J. 1085

Thoracopagus twins 924

Thymic extract 797

Thymic hormone 803

Thymus 803, 1237

Thyroid 230

Thyrotropin-releasing hormone 623

Tietze, F. 1350

Tillman, W. 152

Tina, L. U. 774

Tingle, A. J. 1323

Tolazoline 942

Tolbutamide 1375

Tono-Oka, T. 148

Total parenteral nutrition 932

Trachea 131

Tracheal explant bioassay 31

Trainin, N. 803

$\mathrm{Na}$ transport 1240

$\mathrm{Na}^{+}$transport 1156

Transport 236

Traver, D. 1285

Tremaine, L. M. 1109

Trigeminal airstream stimulation 184

Trypsin 969 
Trypsin-binding Ig 1030

Tsang, R. C. 121, 208

Tsao, F. H. C. 997

Tucker, R. D. 1371

Tuggle, B. 599

Tully, G. W. 1078

Turowski, G. 879

Type II pneumocyte 1097

Type J receptors 1271

Tyrosine 894, 1010

Ueda, K. 44

Uehara, H. 148

Urea cycle 1134

Urea cycle disorder 807

Urinary aldosterone 755

Urinary excretion of polyamines 1137

Urine 1280

Uthne, K. 194

Utter, M. F. 38, 109

Vagal activity 261

Vagotomy 1271

Vagus nerve 611

Valk, C. 1177

Vallota, E. H. 1043

Vallotton, M. B. 742

Vanadium 959

Van Den Berghe, H. 1384

van Leuven, F. 1384

van Stekelenburg, G. J. 1177 van Wijngaarden-Penlerman, M. J. G. 1177

van Zanen, G. E. 1247

Varga, F. 817

Vasopressin 742

Ventilatory response 875

Venous admixture 1167

Ventricular compliance 910

Verdugo, P. 131

Verellen, G. 1065

Verity, M. A. 827

Vida, L. N. 1109

Vincenzi, F. F. 131

Virus 937

Vitamin D toxicity 121

Vitamin K deficiency 1262

Volpe, J. J. 1121

Wagner, D. 152

Walker, W. A. 1290

Wallis, P. 1182

Walters, L. B. 700

Warne, G. L. 211

Warshaw, J. B. 623

Watanabe, K. 1222

Water metabolism 257

Water reabsorption 1172

Watson, B. M. 1150

Weaver, R. L. 184

Weinberg, J. A. 257

Weinberg, M. B., 38

Weinstein, M. M. 230
Weiss, L. S. 1125

Weitzman, R. E. 257, 606, 841

Welliver, R. C. 1015

Whiteside, D. A. 821

Wieke, H. 875

Wieland, P. 963

Wieser, P. B. 10, 626

Will, P. C. 1129

Wilson, C. M. 1319

Wilson, G. B. 1079

Wingert, W. 1015

Winkelstein, J. A. 1093

Winter, J. S. D. 211

Wintour, E. M. 265

Wirjadi, D. 1307

Wolf, B. 1275

Wolf, R. O. 1076

Wong, P. W. K. 1141

Wood, R. E. 1085

Wright, V. J. 1323

Yamamoto, J. 1134

Yelich, M. R. 1217

Yeung, H. N. 821

Young, I. R. 1078

Zachman, R. D. 997

Zijlstra, W. G. 1072

Zimmerly, J. G. 701

Zinc 959 